\title{
Systemic Lupus Erythematosus (SLE): A review on the prevalence, clinical manifestation, and disease assessment
}

\author{
Malarvili Selvaraja ${ }^{1}$, Maha Abdullah², Anim Md Shah ${ }^{3}$, Masita Arip ${ }^{4}$, Syafinaz Amin Nordin ${ }^{1 *}$ \\ ${ }^{1}$ Department of Medical Microbiology \& Parasitology, Faculty of Medicine and Health Sciences, Universiti Putra Malaysia \\ (UPM), Serdang, Selangor, Malaysia \\ ${ }^{2}$ Department of Pathology, Faculty of Medicine and Health Sciences, Universiti Putra Malaysia (UPM), Serdang, Selangor, \\ Malaysia \\ ${ }^{3}$ Department of Medicine, Faculty of Medicine and Health Sciences, Universiti Putra Malaysia (UPM), Serdang, Selangor, \\ Malaysia \\ ${ }^{4}$ Allergy and Immunology Research Centre, Institute for Medical Research, Jalan Pahang, Kuala Lumpur, Wilayah \\ Persekutuan Kuala Lumpur, Malaysia
}

\begin{abstract}
Systemic Lupus Erythematosus (SLE) is a chronic autoimmune disease with a varying clinical phenotype. This disease occurs when the body's tissues are attacked by its own immune system. The aetiology of SLE is not fully understood, but both genetic predisposition and ecological triggers are thought to be involved in the disease manifestation. The study on the prevalence of SLE allows us to identify potential risk factors associated with the disease for the disease and allows proper management and treatment in response to overall disease burden. Hence, this review aims to discuss the prevalence, mortality, clinical manifestation and disease assessment of SLE.
\end{abstract}

Keywords: Systemic Lupus Erythematosus; autoimmune; aetiology; mortality; assessment

Received: $31^{\text {st }}$ July 2020

Accepted: $1^{\text {st }}$ September 2020

Published Online: $05^{\text {th }}$ September 2020

*Correspondence: Syafinaz Amin Nordin, Department of Medical Microbiology \& Parasitology, Faculty of Medicine and Health Sciences, Universiti Putra Malaysia (UPM), Serdang, Selangor, Malaysia; syafinaz@upm.edu.my

Citation: Selvaraja M, Abdullah M, Md Shah A, et al. Systematic Lupus Erythematosus (SLE): A review on the prevalence, clinical manifestation, and disease assessment. Prog Microbes Mol Biol 2020; 3(1): a0000106. https://doi.org/10.3687/ pmmb.a0000106

\section{Introduction}

Systemic Lupus Erythematosus (SLE) is a multisystemic autoimmune disease which can cause chronic inflammation and damage to tissue and almost every organs in our human body ${ }^{[1,2]}$. The exact aetiology of SLE remains unknown, however, it has been hypothesized that genetic, environmental, and other hormonal factors are likely to play a vital role in the occurrence of SLE ${ }^{[3]}$. There are various immunological faults that have been described in the development of SLE which include T- and B-Cell abnormalities, and the failure to clear autoantibodies that leads to generation of immune complexes ${ }^{[4]}$. In addition, an association between SLE disease onset and age, sex, geography and race have been also reported.

SLE affects women more commonly than men with a ratio of $9: 1^{[5]}$. However, male patients tend to have more severe disease manifestation than the female patients ${ }^{[6]}$. It is because the occurrence of lupus nephritis among men is more than women and more likely to develop to end-stage renal disease (ESRD) than women ${ }^{[7]}$. SLE could occur at any age, however, more prevalent in people between the ages of 10 and 50. In terms of ethnicity, the disease affects African American, Asians, Hispanic and Native Americans more frequently than other races in the world. This could be due to genetic and geographical influences which are thought to play a role in the development of SLE. The overall survival rates for 5-year and 10-year was recorded as $82 \%$ and $70 \%$, respectively, whereas survival for 1 -year was $93 \%{ }^{[8]}$. On the other hand, the overall mortality rate among SLE patients was reported as $20.2 \%{ }^{[9]}$. The cause of mortality among SLE patients differs from one to another individual, due to demographical changes including country or region of patient's origin, age, gender and ethnicities.

The exact cause/aetiology of SLE remains elusive, however, the fundamental defect in SLE clearly elucidate the presence of various autoantibodies against self-constituent due to failure in mechanisms that maintain self-tolerance ${ }^{[10]}$. In recent years, the mortality rate of SLE has been declining which possibly due to early detection and pharmacological advances in controlling and managing the disease progression. This review aims to discuss the prevalence, mortality, clinical manifestation and disease assessment of SLE. 


\section{Prevalence of systemic lupus erythematosus}

Worldwide, the disease affects a minimum of 5 million people. The current prevalence of SLE is 20 to 150 cases per 100,000 people ${ }^{[11]}$. In the United States (US), the prevalence of SLE among the Caucasians is approximately 51 per $100,000^{[12]}$. The prevalence of SLE amongst the African-American women is three times higher than the Caucasian women ${ }^{[13]}$. Based on a study in the US, the prevalence of definite SLE within the US community is 54.3 per 100,000 people whereas the suspected cases of SLE is 108.6 per100,000 people ${ }^{[14]}$. It is reported that the prevalence of SLE among AfricanAmerican women was 286 per 100,000 people, a figure nearly twice as high as the prevalence among white woman $^{[15]}$. The incidence rates reported in North America and South America range from 2 to 8 cases per 100,000 people per year. One study by Naleway and colleagues reported the incidence of SLE amongst the US population is 5.1 per $100,000-1.9$ per 100,000 in adult men and 8.2 per 100,00 in adult women ${ }^{[16]}$. Previous study reported an overall incidence of SLE amongst the US community was 5.56 per 100,000 people ${ }^{[17]}$.

Looking into the European Continent, an epidemiological research reported an increase in SLE prevalence from 64.99 per 100,000 people in 1999 to 97.04 per 100,000 people in $2012^{[18]}$. The incidence of SLE during the same study period was 4.91 per 100,000 people with a yearly decline of $1.8 \%$. Approximately, $60 \%$ of SLE populations were women. The highest incidence was reported between the ages of 50 and 59. In terms of ethnicity, Africo-Caribbean people had 2.3-fold higher rate of prevalence and incidence which could be due to regional variation ${ }^{[18]}$. In terms of gender, the females had 10 -fold higher prevalence than the males. The same study also stated that the Africo-Caribbean SLE population had a higher tendency to develop complication such as renal disease and end-stage renal disease (ESRD) approximately $40.5 \%$ and $15.3 \%$ respectively as compared to Caucasians in the same SLE population whose proportions were only of $18.8 \%$ and $4.5 \%$ respectively ${ }^{[18]}$. In another study, it was reported that the Caucasians of England origin had a lower prevalence compared to Afro-Caribbean, Hispanics and Asians ${ }^{[19]}$. In addition to the above, Table 1 shows the prevalence rate for other countries in the European Continent.

Table 1. Summary of SLE disease prevalence for European states.

\begin{tabular}{|l|l|c|}
\hline Country & Prevalence & Reference \\
\hline Lithuania & $16.2 / 100,000(0.016 \%)$ & {$[20]$} \\
\hline South Ireland & $\begin{array}{l}21.7-39.5 / 100,000 \\
(0.022 \%-0.039 \%)\end{array}$ & {$[21]$} \\
\hline Norway & $\begin{array}{l}47.6-57.9 / 100,000 \\
(0.048 \%-0.058 \%)\end{array}$ & {$[22]$} \\
\hline
\end{tabular}

For Oceanic continent, looking at Australia, although there is a lack of published information on SLE, one identified study has reported that two ethnic groups, namely Aboriginal Australian and Caucasians were known to have SLE. Whereas, a study reported in 1998 stated that the prevalence of SLE in Queensland, Australia was 45.3 per 100,000 people ${ }^{[23]}$. In another study conducted at the central part of Australia from the period of 19961997, the prevalence of SLE was 92.8 per 100,000 people among the Aborigines ethnic group ${ }^{[24]}$. Besides that, one study carried out at the Australia Northern territory from the period of 1986-1990, reported SLE prevalence of 19.3 per 100,000 and 52.6 per 100,000 people amongst the Australian Caucasians and Aborigines community respectively. Another study reported the incidence of SLE amongst Aborigines people was 11 per 100,000 people at the Northern Territory of Australia ${ }^{[25]}$.

A study based in Canada reported that the prevalence of SLE in Canada falls within the range of 22.1 and 51.0 cases per 100,000 people ${ }^{[26,27]}$. In addition to that, another study reported that the disease generally affects $0.05 \%$ of Canadian adults and it is 10 times more prevalent amongst women than men ${ }^{[28]}$. One study from Brazil population showed that the prevalence of SLE is 3 per 100,000 people with predominantly women ${ }^{[29]}$. Similarly, another study in Brazil reported SLE prevalence of 20 to 150 cases per 100,000 people $^{[30]}$. In Saudi Arabia, the prevalence of SLE amongst its nationals was estimated at 19.28 per 100,000 people ${ }^{[31]}$.

Amongst Asians, SLE prevalence has been estimated to be between 30 and 50 per 100,000 individuals. It has been reported that SLE is more common among the Chinese population in $\mathrm{Asia}^{[32]}$. Another study reported three cases of SLE out of 4192 adults registered for rheumatic disorder in the north of China (Beijing), and one out of 5057in the south of China (Shantou) from Han population, to have SLE disease ${ }^{[33]}$. Similarly, the prevalence of SLE amongst the Han population in China was estimated at 37 to 70 cases per 100,000 people $^{[34]}$. These data show the Chinese population has a higher prevalence of rheumatic disease including SLE in comparison to the Caucasians. In Taiwan, the prevalence of SLE among paediatric population was reported at 6.3 per 100,000 people. The prevalence in girls were 11.2 cases per 100,000 , which is 6.2 times higher than that in boys $(1.8 \text { cases per } 100,000)^{[35]}$.

In India, a study conducted in Northern India demonstrated a prevalence of 3.2 per 100,000 people with SLE, which is at lowest compared to other Asian countries ${ }^{[36,37]}$. Moreover, studies also reported that there were no SLE cases found amongst adults from certain rural and urban areas of Jammu, India ${ }^{[38]}$. Amongst Malaysian populations, consisting of three major ethnic groups; Malays (55.1\%), Chinese (24.3\%) and Indians (7.4\%) out of a total population of 22 millions of people, SLE prevalence has been reported to be 43 per 100,000 people ${ }^{[37]}$. Amongst the three communities, Chinese population represents the highest prevalence rate of SLE of 57 per 100,000 followed by Malays 33 per 100,000 and Indians with only 14 per 100,000 people.

\section{Survival and mortality of SLE}

Generally, the survival rates of SLE can be categorized into three groups. The categories are survival rates at 1 year, 5 years and 10 years. The overall survival rate at 1 year fall 
within 93 to $98 \%$, followed by at 5 years ranging from 60 to $97 \%$ and 70 to $94 \%$ at 10 years. Moreover, with the advancement in pharmacological therapy and treatment, the overall survival rate of SLE is expected to improve.

The cause of mortality amongst SLE patients varies from one individual to another partly due to demographical variances. The demographical differences include geographical origin of patients, age, gender and ethnicity. These factors are considered crucial as some studies have proven that they have major roles in the pathogenesis and survival of SLE. Socioeconomic status often determined by occupation and residing area of patients have also been found to effect survivability and management of SLE disease ${ }^{[39,40]}$. Besides that, the disease duration also determines the mortality rate amongst the patients.

Several studies have reported that generally, active disease and multiple kind of infections are the most common factors leading to early death among SLE patients. During active phase of SLE, patients receive numerous immunosuppressant which may have negative impact on their natural immune system, thus paving the way for opportunistic infections and even death in severe cases. On the other hand, death in later stage of SLE is usually related to vascular events such as cardiovascular disease $^{[41]}$, thrombotic events ${ }^{[42]}$, non-Hodgkin lymphoma, lung cancer and renal disorders such as lupus nephritis ${ }^{[26]}$. However, these underlying causes of death vary according to geographical location and ethnicity. For instance, amongst the US populations, the African-American, American Indians and the Asians have higher mortality rate compared to the Caucasians ${ }^{[41]}$. It was also found that the causes of death amongst the younger and elderly patients were mainly due to infections in the former and cardiovascular or renal complications in the latter.

Another study conducted in the US reported the SLE mortality rate is higher among the African Americans, American Indians and Asians compared to the Americans ${ }^{[41]}$. It is noted that ethnicity and socioeconomic could be the possible cause of higher mortality rate among the African Americans. Based on medical intervention and clinicians, infections are the main cause of death among the younger patients, whereas cardiovascular or renal complications are the clinical manifestation among the elderly group.

A study conducted in Malaysia on the mortality patterns amongst Malaysian SLE patients, reported that 30\% of patients died of infection, followed by $15 \%$ of renal disorders, $14 \%$ of pulmonary disorders, $7 \%$ of cardiovascular diseases, $5 \%$ of central nervous system, $1 \%$ of malignancy, $1 \%$ for acute anaphylaxis, $27 \%$ of unknown reason and remaining $19 \%$ died due to SLE as a contributory factor of death. The study also mentioned that the Chinese community represents the majority of those who live with SLE at approximately $73 \%$, followed by Malays at $18 \%$ and Indians the least at $9 \%$. The average age at death reported was 28.6 years $^{[43]}$.

\section{Clinical manifestation of SLE}

Systemic lupus erythematosus is known to be a gradually worsening multi-systemic disease with mild signs and lesser number of affected systems in the beginning stage. After some years or with a rapidly progressive condition, over a few weeks or months, it continues to involve and affect more systems severely. Over time, there will be various clinical and laboratory manifestations. Autoantibodies are the hallmark of laboratory manifestation for SLE diagnosis. Fatigue is the one of the most common complaints of SLE patients. However, it has a broad association with many other diseases such as fibromyalgia, hypothyroidism, depression, anaemia, pulmonary or cardiovascular diseases. Moreover, some other intrinsic signs of an active lupus that may commonly occur amongst the patients include fever, anorexia, lymphadenopathy and weight loss. However, all of the above symptoms cannot be attributed to lupus alone until infection and malignancy causes are ruled out. The stage of involvement and severity based on every organ system is further elucidated as below.

\section{Mucocutaneous manifestations / Dermatological Features}

Dermatological features amongst SLE patients account for four out of 11 revised criteria based on the SLE classification tool portrayed in The American College of Rheumatology (ACR). The skin involvement is claimed to be as high as $85 \%$ among the SLE patients ${ }^{[44]}$. One of the most commonly identified signs amongst the SLE patients are the photosensitivity rashes and the butterfly or malar rash on the face. Other familiar mucocutaneous signs in SLE patients include mouth ulcers and alopecia or hair loss. Hair loss can be categorized into three types which include localized diffused alopecia, "frizz" or frontal hair loss and severe diffused alopecia with minimal sign of new hair growth ${ }^{[45]}$. Discoid lesion (DL) and maculopapular lesion, splinter haemorrhages, dilated capillaries at the nail base, bullous lesions, angioneurotic oedema and livedo reticularis are also some of the identified dermatological manifestations ${ }^{[46]}$. The discoid lesion repeatedly leads to scarring and older lesions may result in pigmentary changes, either hypopigmentation or hyperpigmentation. Alopecia scaring may appear in relation to discoid rash on the scalp region. Ulcers in mouth, nasal and genital have also been associated with SLE but they are less common. Raynaud's phenomenon is one of the mildly described signs amongst SLE patients. It can be linked to severe digital ischaemia and maturation of gangrene. Raynaud's is a disease affecting the blood vessels at the fingers and toes. It will make blood vessels to be narrowed around the area in cold or stress conditions. This feature is difficult to be recognized amongst the dark skinned individuals unless there is sparing of some fingers ${ }^{[47]}$. Vasculitis is usually identified at the nail folds and finger tips ${ }^{[44]}$. When it occurs, it may develop into a tender, deep, frank ulceration which can either take months to heal or lead to secondary infections. The other more frequently found lesion is shingles, which caused by herpes zoster and often occurs in patients taking immunosuppressant. Maculopapular rash is a type of rash that appears flat and red on the skin and covered with small confluent bumps which can become infected in the SLE patients.

\section{Musculoskeletal Involvement}

Arthralgia is defined as an inflammatory joint pain which develops along with morning stiffness or gelling that 
happens after a period of rest. It occurs approximately in $90 \%$ of SLE patients ${ }^{[48]}$. Synovial effusion is less common. However, only small volume is present even if it happens. In addition, non-erosive arthritis with joint tenderness and swelling may also form ${ }^{[49]}$. About $10 \%$ of SLE patients have been found to develop Jaccoud's arthropathy, whereas among rheumatoid arthritis patients the deformities occur along with joint erosions. It has also been pointed out that SLE patients may develop osteoarthritis as they age. Bouchard's nodes that occur at the proximal inter phalangeal joints and Heberden's nodes that occur at the distal interphalangeal joints caused by osteophytes in osteoarthritis is different from acute synovitis that occurs due to lupus flare. Tenosynovitis is an early symptom of SLE associated with tendon rupture found on patellar tendon, Achilles tendon, the long head of the biceps, the triceps and the extensor tendons of the hands. It has been estimated that the, muscle involvement among SLE patients is up to $30-50 \%$ of patients ${ }^{[50]}$.

\section{Respiratory/ Lung Involvement}

Lung is one of the vital organs in human body and it is highly susceptible to secondary infections caused by bacteria, viruses and fungi which may lead to pneumonia in SLE patients. Pain on deep inspiration is one of the frequent complains amongst SLE patients which is caused by pleurisy. Generally, the immunosuppressive agents taken by the SLE patients may result in an immunosuppression which may trigger infections. Pleural effusion is a sign found in approximately half of the SLE patients specifically during disease flares. Pulmonary fibrosis, pulmonary haemorrhage, oedema and pulmonary embolism caused by lupus pneumonitis are other rare manifestations ${ }^{[51]}$.

\section{Cardiovascular Features}

The most prevalent cardiovascular sign in SLE is chest pain. A pericardial rub is more common than a significant pericardial effusion amongst SLE patients. Myocardial involvement in SLE is less common than pericardial disorder. Studies have reported that arthrosclerosis increases the risk of cardiovascular events amongst SLE patients. Systolic murmurs are also commonly found sign, affecting $30 \%$ of SLE patients, whereas diastolic murmurs are less common ${ }^{[52,53]}$.

\section{Neuropsychiatric Features}

SLE can affect both the central and peripheral nervous systems. Some of the most frequently observed central nervous system features are headache (benign intracranial, hypertension), seizure disorders, psychosis, myelopathy, movement disorders, mood disorder, demyelinating syndrome, cognitive dysfunction, cerebrovascular disease, anxiety disorder, aseptic meningitis and delirium ${ }^{[54]}$. At some instance, drugs could possible precipitate some of the above-mentioned conditions. Steroids are known to induce psychosis in some SLE patients. Acute inflammatory demyelinating polyradiculoneuropathy (Guillain-Barre-Syndrome), autonomic disorder, mononeuropathy (single or multiplex), myasthesia gravis, neuropathy (cranial) and plexopathy are some of the manifestations involving peripheral nervous system ${ }^{[55]}$. Severe neuropsychiatric condition of lupus is known as neuropsychiatric SLE (NP-SLE), the third leading cause of death amongst SLE patients $^{[56]}$.

\section{Gastrointestinal Involvement}

Various non-specific gastrointestinal manifestations have been reported in SLE patients. Nausea is one of the common symptoms followed by abdominal pain in SLE patients. Vomiting and diarrhoea are less common symptoms but may occur with active SLE. When perforation occurs, necrotizing vasculitis can be seen pathologically. Ascites, dysphagia and pancreatitis are other rare gastrointestinal manifestations $^{[57]}$.

\section{Ophthalmic Involvement}

The recurrent factors that leads to red eye in lupus are episcleritis and sicca (dry eye). Approximately $8 \%$ of SLE patients develop inflammation of the retinal artery during the early days of disease course. Retinal vasculitis is an active systemic disorder which can lead to visual loss besides optic neuritis. Uveitis is less common and affects less than $1 \%$ of patients. Vaso-occlusive disorder that affects the retina or choroidal vessels and causes anterior ischaemic optic neuropathy may also lead to a vision loss. Myositis of eye muscles that lead to diplopia and / or proptosis is a rare occurrence amongst SLE patients ${ }^{[58]}$.

\section{Haematological Abnormalities}

It is very common among SLE patients to have haematological abnormalities such as anaemia, thrombocytopenia, and leukopenia during the course of disease ${ }^{[59]}$. Autoimmune haemolytic anaemia in SLE is another distinguishing symptom amongst SLE patients reflecting decreased level of serum iron and iron binding capacity. Multiple mechanisms lead to iron deficiency which include excessive usage of steroidal and non-steroidal anti-inflammatory drugs resulting in gastrointestinal bleeding ${ }^{[57]}$. Renal insufficiency, blood loss, dietary insufficiency and infections may lead to anaemia. Another persistent and typical feature of SLE is leucopoenia $\left(<4.0 \times 10^{9} / \mathrm{L}\right)$ which is found in over $90 \%$ of SLE patients. Autoantibody deposition that diminishes the function of immune cells and compliment activation is also partly involved in the development of haematological abnormalities amongst SLE patients. Moreover, immunosuppressive agents also contribute to severe leucopoenia ${ }^{[60]}$. Thrombocytopenia (platelet count $<100 \times 10^{9} / \mathrm{L}$ ) is one of the common laboratory findings in SLE patients. It can appear in either chronic or acute form. Chronic form is related to mild disease, whereas acute form is similar to idiopathic autoimmune thrombocytopenic purpura. Platelet dissociation is known to be directed by anti-platelet antibodies (APL). APL is also associated with thrombocytopenia and thrombosis ${ }^{[61]}$.

\section{Renal Involvement}

More than $70 \%$ of patients with SLE have been estimated to have Lupus Nephritis (LN) at some stage of their disease progression. The World Health Organization 
(WHO) classification for Lupus Nephritis has been published to facilitate a more accurate description of renal histopathological biopsies by the International Society of Nephrology and the Renal Pathology Society (Table 2) ${ }^{[62]}$.

Table 2. Classification of lupus nephritis.

\begin{tabular}{|c|c|}
\hline Classes & Description \\
\hline Minimal mesangial lupus nephritis & $\begin{array}{l}\text { Normal glomeruli at light microscopy } \\
\text { Mesangial immune deposits on immunofluorescence }\end{array}$ \\
\hline Mesangial proliferative lupus nephritis & $\begin{array}{l}\text { Mesangial hyper-cellularity or expansion with mesangial immune deposits in light microscopy } \\
\text { Some subepithelial or subendothelial deposits on immunofluorescence or electron microscopy }\end{array}$ \\
\hline Focal lupus nephritis & $\begin{array}{l}\text { Involves less than } 50 \% \text { glomeruli } \\
\text { Active or inactive lesions with subendothelial deposits }\end{array}$ \\
\hline Diffuse lupus nephritis & $\begin{array}{l}\text { Involves more than } 50 \% \text { glomeruli } \\
\text { Active or inactive diffuse, segmental, or global endo- or extracapillary glomerulonephritis with } \\
\text { subendothelial deposits. } \\
\text { Divided into diffuse segmental when }<50 \% \text { of involved glomeruli have segmental lesions and diffuse } \\
\text { global when }>50 \% \text { of involved glomeruli have global lesions }\end{array}$ \\
\hline Membranous lupus nephritis & $\begin{array}{l}\text { Global or segmental subepithelial immune deposits by light microscopy and immunofluorescence or } \\
\text { electron microscopy, with or without mesangial changes. } \\
\text { Class V lupus nephritis may occur in combination with class III or class IV disease, in which case both } \\
\text { are diagnosed. } \\
\text { Class V disease may show advanced sclerosis }\end{array}$ \\
\hline Advanced sclerosis lupus & $>90 \%$ of glomeruli scleroses without residual activity \\
\hline
\end{tabular}

\section{Obstetric-related Issues}

Although, SLE is not directly associated with infertility, it has been associated with pregnancy, particularly in the second or third trimester which is caused by lupus flare. Moreover, in SLE patients who develop active Lupus Nephritis (LN) during conception, either as new onset or flare, increases the risk of preterm delivery, preeclampsia, maternal mortality, foetal / neonatal demise and intrauterine growth restrictions. Other common conditions in SLE pregnant women are cutaneous disease (25-90\%), arthritis $(20 \%)$ and haematological involvement which include thrombocytopenia $(10-14 \%)^{[63]}$.

\section{Laboratory Criteria}

The evolution of autoantibodies in human body has been associated with the manifestation of SLE disease. One of the classic, non-specific antibodies that are present in approximately $96 \%$ of SLE patients is anti-nuclear antibodies (ANAs) (Manson and Isenberg, 2003). ANAs are antibodies or immunoglobulins that bind to one or more antigens expressed within the nucleus of human cells in relation to connective tissue disease or infections. Anti-double stranded DNA (AdsDNA) are more specific anti-nuclear antibodies that only target double stranded DNA. Studies have reported that anti-dsDNA antibodies have a strong link with glomerulonephritis in SLE patients. Anti-dsDNA is also used for diagnosis purpose and to monitor the disease progression and anti-Smith (anti-Sm) antibodies ${ }^{[4]}$. Anti-Smith (anti-Sm) antibodies identify extractable nuclear antigens (anti-ENA) which include anti-Ro and anti-La antibodies. Other antibodies that have been found to be associated with SLE disease include antiRNP antibodies, Rheumatoid factor, IgG anticardiolipin antibodies, IgM anti cardiolipin antibodies and lupus anti-coagulant. Moreover, complement activation plays a vital role in the deposition of immune complexes when autoantibodies bind to their target antigens. Low level of complements, C3 and C4 are highly associated with lupus nephritis and vasculitis ${ }^{[64]}$.

\section{Non-Specific Features}

Lymphadenopathy is defined by changes in number, characteristics or size of the lymph node. In SLE, this is a benign condition which can be diagnosed at any stage of the disease development. Studies have 1 reported that this sign is commonly found in younger patients with cutaneous involvement ${ }^{[65]}$. Other sign including spleen enlargement or known as splenomegaly is estimated to happen in $10 \%$ of SLE patients.

\section{The disease assessment}

One of the challenging and difficult aspects in SLE is measuring the disease activity due to the complexity of disease nature which affecting multiple organs and the clinical outcomes from one to another SLE patient. Number of studies attempted in order to find definition for disease activity in SLE and how it should be measured. Measuring the disease activity in SLE is one of the three domains of SLE assessments that include the measuring of damages caused by SLE and the quality of life ${ }^{[66]}$.

Generally, corticosteroids and immunosuppressive that is used in controlling SLE symptoms plays a vital role in disease activity. This is due to their pharmacological properties producing various side effects such as diabetes, 
osteoporosis, arterial hypertension and neoplasia among SLE patients. Various indices or disease assessment tools were discovered with the objective standardizing SLE activity assessment. The commonly used indices are as follows: LAI (Lupus Activity Index); SLAM (Systemic Lupus Activity Measure); ECLAM (European Consensus Lupus Activity Measurement); BILAG (British Isles Lupus Assessment), SLEDAI (Systemic Lupus Erythematosus Disease Activity Index); SELENASLEDAI (Safety of Estrogen in Lupus Erythematosus National Assessment-SLEDAI) and SLEDAI-2KG ${ }^{[67]}$.

Among the few listed instruments above, SLEDAI that been introduced in 1985 was proven to be the most reliable and reproducible apart from being sensitive to change in a patient condition when used by various investigators. It is an index that measures disease activity by considering the organ affected and includes 24 clinical and laboratory parameters of nine organ systems in its evaluation. SLEDAI assesses disease activity in the previous 10 days. It assesses 16 clinical features and 8 laboratory indices. The scores of the descriptors range from 1 to 8 . Active disease indicated by scores greater than 8 and the total possible score for all 24 descriptors is $105^{[68]}$. Later SLEDAI-2K was introduced in 2002, a modified version of original SLEDAI but there was one limitation. This revised index does not account for severity within each descriptor ${ }^{[69]}$ América The Systemic Lupus Activity Measure-revised, the Mexican Systemic Lupus Erythematosus Disease Activity Index (SLEDAI. However, it was proven to have best discriminative validity in which able to differentiate active patients from inactive ones and lowest cost. Later another novel tool was developed in order to measure lupus disease activity index known as SLEDAI-2K GCS (SLEDAI-2KG) which outlined disease activity while considering on glucocorticosteroid (GCS) dose and having same descriptors as SLEDAI-2K but with different weight scores based on the dose of $\mathrm{GCS}^{[70]}$.

SLAM (systemic lupus activity measure), was first reported in 1986 and later revised in 2001 based on consensus of the lupus council of the American College of Rheumatology ${ }^{[71]}$. The difference between SLAM and SLEDAI is illustrated in Table 3. SLAM measured the disease severity in the domains of 9 organ systems and 7 laboratory measures as follows; constitutional, integument, eye, reticuloendothelial, gastrointestinal, cardiovascular, pulmonary, neuromotor, joints and laboratory parameters. The total SLAM score ranges from 0-84, with each organ items scored $0-3$ points manifested within one month (30 days) period, in which severity having highest score by item $^{[72]}$.

Table 3. Difference between SLEDAI and SLAM scoring system.

\begin{tabular}{|l|l|}
\hline \multicolumn{1}{|c|}{ SLEDAI } & SLAM \\
\hline$\square \quad$ Reliable, validated, sensitive and responsiveness to adapt with time & $\square \quad$ Reliable, validated, sensitive and responsiveness change over time \\
$\square \quad$ Very practical and widely used for clinical and research purpose. & $\square \quad$ Measures disease severity within one month \\
$\square \quad$ Scored within last days if present with symptoms & $\square \quad$ Laboratory studies needed \\
$\square \quad \begin{array}{l}\text { None of the SLEDAI version captures on improving or worsening } \\
\text { and do not include severity within an organ system. Thus, this is less } \\
\text { sensitive to compared to other instruments }{ }^{[73]}\end{array}$ & $\square \quad$ Responsiveness to patient care: highly responsive \\
$\square \quad$ Laboratory information required. & \\
\hline$\quad$ Responsiveness to patient care ${ }^{[69]}$ & \\
\hline
\end{tabular}

\section{Conclusion}

Systemic lupus erythematosus (SLE) is an autoimmune disease that effects the quality of life for many people. This disease gradually worsen over the time and patients are on medications for life long. Moreover, the disease manifestation and complications vary from one to another individual, taking into the consideration of their ethnicity and geographical region where they are origin. It is a daunting task to determine the cause of the disease in one population. Hence, continuous monitoring and surveillance is required to manage the disease progression.

\section{Author Contribution}

The literature search and manuscript writing were performed by MS. MA, AM-S and MA provided vital guidance and support as content expert and proofread of the writing. The project was founded by SA-N.

\section{Conflict of Interest}

All the authors declare that the research was conducted in the absence of any commercial or financial relationships that could be construed as a potential conflict of interest. 


\section{Acknowledgements}

This study was supported by funding from the Ministry of Higher Education, Malaysia, FRGS/2/2013/SKK01/ UPM/02/8, with the Grant No. 5524400. We would like to thank the Director General (DG) of Health Malaysia for his permission to publish this paper. We would also like to thank all staff in the Nephrology Unit, Hospital Serdang; and Immunology Unit, Department of Pathology, Faculty of Medicine and Health Sciences, Universiti Putra Malaysia for their excellent assistance throughout this research.

\section{Reference}

1. Islam MA, Khandker SS, Alam SS, et al. Vitamin D status in patients with systemic lupus erythematosus (SLE): A systematic review and meta-analysis. Autoimmun Rev 2019; 18(11): 102392

2. Selvaraja M, Abdullah M, Arip M, et al. Elevated interleukin-25 and its association to Th2 cytokines in systemic lupus erythematosus with lupus nephritis. PLoS One 2019; 14(11): e0224707.

3. Rosser EC ans Mauri C. A clinical update on the significance of the gut microbiota in systemic autoimmunity. J Autoimmun 2016; 74: 85-93. Mok C and Lau C. Pathogenesis of systemic lupus erythematosus. J Clin Pathol 2003; 56(7): 481- $\square 490$.

5. Tedeschi SK, Bermas B, and Costenbader KH. Sexual disparities in the incidence and course of SLE and RA. Clin Immunol 2013; 149(2): 211-218.

6. Schwartzman-Morris $\mathrm{J}$ and Putterman C. Gender differences in the pathogenesis and outcome of lupus and of lupus nephritis. Clin Dev Immunol 2012; 2012 .

7. Nowling TK and Gilkeson GS. Mechanisms of tissue injury in lupus nephritis. Arthrit Res Ther 2011; 13(6): 1-9.

8. Paton N, Cheong I, Kong N, et al. Risk factors for infection in Malaysian patients with systemic lupus erythematosus. QJM 1996; 89(7): 531-538.

9. Wang F, Wang CL, Tan CT, et al. Systemic lupus erythematosus in Malaysia: a study of 539 patients and comparison of prevalence and disease expression in different racial and gender groups. LUPUS 1997; 6(3): 248-253.

10. Kumar K, Kole AK, Karmakar PS, et al. The spectrum of thyroid disorders in systemic lupus erythematosus. Rheumatol Int 2012; 32(1): 73-78.

11. Pons-Estel GJ, Alarcón GS, Scofield L, et al. Understanding the epidemiology and progression of systemic lupus erythematosus. Semin Arthritis Rheum 2010; 39(4): 257-268.

12. Cervera R, Khamashta MA, Font J, et al. Morbidity and mortality in systemic lupus erythematosus during a 10-year period: a comparison of early and late manifestations in a cohort of 1,000 patients. Medicine 2003; 82(5): 299-308.

13. Borchers AT, Naguwa SM, Shoenfeld Y, et al. The geoepidemiology of systemic lupus erythematosus. Autoimmun Rev 2010; 9(5): A277A287.

14. Helmick CG, Felson DT, Lawrence RC, et al. Estimates of the prevalence of arthritis and other rheumatic conditions in the United States: Part I. Arthritis Rheum 2008; 58(1): 15-25.

15. Feldman CH, Hiraki LT, Liu J, et al. Epidemiology and sociodemographics of systemic lupus erythematosus and lupus nephritis among US adults with Medicaid coverage, 2000-2004. Arthritis Rheum 2013; 65(3): 753-763.

16. Naleway A, Davis M, Greenlee R, et al. Epidemiology of systemic lupus erythematosus in rural Wisconsin. LUPUS 2005; 14(10): 862866.

17. Uramoto KM, Michet Jr CJ, Thumboo J, et al. Trends in the incidence and mortality of systemic lupus erythematosus, 1950-1992. Arthritis Rheum 1999; 42(1): 46-50.

18. Rees F, Doherty M, Grainge M, et al. The incidence and prevalence of systemic lupus erythematosus in the UK, 1999-2012. Ann Rheum Dis 2016; 75(1): 136-141.

19. Lau C, Yin G, and Mok M. Ethnic and geographical differences in systemic lupus erythematosus: an overview. LUPUS 2006; 15(11): 715-719.

20. Dadoniene J, Adomaviciute D, Rugiene R, et al. The prevalence of systemic lupus erythematosus in Lithuania: the lowest rate in Northern Europe. LUPUS 2006; 15(8): 544-546.

21. Gourley I, Patterson C, and Bell A. The prevalence of systemic lupus erythematosus in Northern Ireland. LUPUS 1997; 6(4): 399-403.

22. Nossent HC. Systemic lupus erythematosus in the Arctic region of Norway. The Journal of rheumatology 2001; 28(3): 539-546.

23. Bossingham D. Systemic lupus erythematosus in the far north of Queensland. LUPUS 2003; 12(4): 327-331.

24. Segasothy M and Phillips PA. Systemic lupus erythematosus in
Aborigines and Caucasians in central Australia: a comparative study. LUPUS 2001; 10(6): 439-444.

25. Anstey N, Bastian I, Dunckley H, et al. Systemic lupus erythematosus in Australian aborigines: high prevalence, morbidity and mortality. Aust N Z J Med 1993; 23(6): 646-651.

26. Bernatsky S, Joseph L, Pineau C, et al. A population-based assessment of systemic lupus erythematosus incidence and prevalence — results and implications of using administrative data for epidemiological studies. Rheumatology 2007; 46(12): 1814 1818 .

27. Afro-Caribbean S. Systemic lupus erythematosus in North American Indians: a population based study. J Rheumatol 2000 27: 8 .

28. Badley E. Arthritis in Canada: an ongoing challenge. Ottawa: Health Canada 2003

29. Senna ER, De Barros ALP, Silva EO, et al. Prevalence of rheumatic diseases in Brazil: a study using the COPCORD approach. J Rheumatol 2004; 31(3): 594-597.

30. Rother $\mathrm{N}$ and Van der Vlag J. Disturbed $\mathrm{T}$ cell signaling and altered Th17 and regulatory T cell subsets in the pathogenesis of systemic lupus erythematosus. Front Immunol 2015; 6: 610

31. Al-Arfaj AS, Al-Balla SR, Al-Dalaan AN, et al. Prevalence of systemic lupus erythematosus in central Saudi Arabia. Saudi Med J 2002; 23(1): 87.

32. Frank A. Apparent predisposition to systemic lupus erythematosus in Chinese patients in West Malaysia. Ann Rheum Dis 1980; 39(3): 266-269.

33. Wigley R, Zhang N, Zeng Q, et al. Rheumatic diseases in China: ILAR-China study comparing the prevalence of rheumatic symptoms in northern and southern rural populations. J Rheumatol 1994; 21(8): 1484

34. Zeng QY, Chen R, Darmawan J, et al. Rheumatic diseases in China. Arthrit Res Ther 2008; 10(1): R17.

35. Huang J, Yao T, and See L. Prevalence of pediatric systemic lupus erythematosus and juvenile chronic arthritis in a Chinese population: a nation-wide prospective population-based study in Taiwan. Clin Exp Rheumatol 2004; 22(6): 776-780.

36. Malaviya A, Singh R, Singh Y, et al. Prevalence of systemic lupus erythematosus in India. LUPUS 1993; 2(2): 115-118.

37. Osio-Salido E and Manapat-Reyes H. Epidemiology of systemic lupus erythematosus in Asia. LUPUS 2010; 19(12): 1365-1373.

38. Mahajan A, Jasrotia DS, Manhas A, et al. Prevalence of major rheumatic disorders in Jammu. 2003.

39. Krieger N, Williams DR, and Moss NE. Measuring social class in US public health research: concepts, methodologies, and guidelines. Annu Rev Public Health 1997; 18(1): 341-378.

40. Sutcliffe N, Clarke A, Gordon C, et al. The association of socioeconomic status, race, psychosocial factors and outcome in patients with systemic lupus erythematosus. Rheumatology 1999 38(11): 1130-1137.

41. Borchers AT, Keen CL, Shoenfeld Y, et al. Surviving the butterfly and the wolf: mortality trends in systemic lupus erythematosus. Autoimmun Rev 2004; 3(6): 423-453.

42. Cervera R. Systemic lupus erythematosus in Europe at the change of the millennium: lessons from the "Euro-Lupus Project" Autoimmun Rev 2006; 5(3): 180-186.

43. Yeap S, Fauzi A, Kong N, et al. Influences on bone mineral density in Malaysian premenopausal systemic lupus erythematosus patients on corticosteroids. LUPUS 2009; 18(2): 178-181.

44. Uva L, Miguel D, Pinheiro C, et al. Cutaneous manifestations of systemic lupus erythematosus. Autoimmune Dis 2012; 2012

45. Al-Refu K and Goodfield M. Hair follicle stem cells in the pathogenesis of the scarring process in cutaneous lupus erythematosus. Autoimmun Rev 2009; 8(6): 474-477.

46. Parish LC, Kennedy RJ, and Hurley HJ. Palmar lesions in lupus erythematosus. Arch Dermatol 1967; 96(3): 273-276.

47. Wigley FM. Raynaud's phenomenon. New Engl J Med 2002 347(13): 1001-1008

48. Petri M. Musculoskeletal complications of systemic lupus erythematosus in the Hopkins Lupus Cohort: an update. Arthritis Rheum 1995; 8(3): 137-145.

49. Torrente-Segarra V, Monte TCS, and Corominas $\mathrm{H}$ Musculoskeletal involvement and ultrasonography update in systemic lupus erythematosus: New insights and review. Eur. J Rheumatol Inflammation 2018; 5(2): 127.

50. Sutrisno RN, Rahmadi AR, Novita N, et al. Most frequen musculoskeletal manifestation of systemic lupus erythematosus patients in Dr. Hasan Sadikin General Hospital Bandung. Indonesian Journal of Rheumatology 2017; 9(2).

51. Holgate S, Glass D, Haslam P, et al. Respiratory involvement in systemic lupus erythematosus. A clinical and immunological study. Clin Exp Immunol 1976; 24(3): 385.

52. Mocarzel L, Lanzieri PG, Montes RA, et al. Systemic lupus erythematosus: review of cardiovascular aspects. International Journal of Cardiovascular Sciences 2015; 28(3): 251-61.

53. Miner JJ and Kim AH. Cardiac manifestations of systemic lupus erythematosus. Rheumatic Diseases Clinics 2014; 40(1): 51-60.

54. Faria R, Goncalves J, and Dias R. Neuropsychiatric systemic lupus erythematosus involvement: towards a tailored approach to our patients? Rambam Maimonides Medical Journal 2017; 8(1).

55. Tanabe J and Weiner MW. MRI $\square$ MRS of the brain in systemic lupus erythematosus: how do we use it to understand causes of 
clinical signs? Ann N Y Acad Sci 1997; 823(1): 169-184

56. Souirti Z, Lahlou M, El Ouali O, et al. Neuropsychiatric systemic lupus erythematosus. 2013.

57. Tian X-P and Zhang X. Gastrointestinal involvement in systemic lupus erythematosus: insight into pathogenesis, diagnosis and treatment. World J Gastroenterol 2010; 16(24): 2971.

58. Sivaraj R, Durrani O, Denniston A, et al. Ocular manifestations of systemic lupus erythematosus. Rheumatology 2007; 46(12): 17571762.

59. Teke HÜ, Cansu DÜ, and Korkmaz C. Detailed features of hematological involvement and medication-induced cytopenia in systemic lupus erythematosus patients: single center results of 221 patients. Eur J Rheumatol Inflammation 2017; 4(2): 87.

60. Fayyaz A, Igoe A, Kurien BT, et al. Haematological manifestations of lupus. Lupus science \& medicine 2015; 2(1)

61. Bashal F. Hematological disorders in patients with systemic lupus erythematosus. Open Rheumatol J 2013; 7: 87.

62. Yap DY and Lai KN. Pathogenesis of renal disease in systemic lupus erythematosus - the role of autoantibodies and lymphocytes subset abnormalities. Int J Mol Sci 2015; 16(4): 7917-7931.

63. Clowse ME. Lupus activity in pregnancy. Rheum Dis Clin North Am 2007; 33(2): 237-252

64. Dumestre-Pérard C, Clavarino G, Colliard S, et al. Antibodies targeting circulating protective molecules in lupus nephritis: Interest as serological biomarkers. Autoimmun Rev 2018; 17(9): 890-899.

65. Rosa Neto NS, Bonfiglioli KR, Milanez FM, et al. Lymphadenopathy and systemic lupus erythematosus. Revista brasileira de reumatologia 2010; 50(1): 96-101.

66. Freire EAM, Souto LM, and Ciconelli RM. Assessment measures in systemic lupus erythematosus. Revista brasileira de reumatologia 2011; 51(1): 75-80

67. Pope JE. Measuring flares in systemic lupus erythematosus. 2014, Oxford University Press.

68. Castrejón I, Tani C, Jolly M, et al. Indices to assess patients with systemic lupus erythematosus in clinical trials, long-term observational studies, and clinical care. Clin Exp Rheumatol 2014; 32(5 Suppl 85): $85-95$.

69. Uribe AG, Vilá LM, McGwin G, et al. The Systemic Lupus Activity Measure-revised, the Mexican Systemic Lupus Erythematosus Disease Activity Index (SLEDAI), and a modified SLEDAI-2K are adequate instruments to measure disease activity in systemic lupus erythematosus. The Journal of rheumatology 2004; 31(10): 1934 1940.

70. Urowitz M, Gladman D, Su J, et al. S1D: 5 Sle disease activity index glucocorticosteroid index (sledai-2kg) identifies more responders than sledai-2k. Archives of Disease in childhood 2018.

71. Saba J, Quinet RJ, Davis WE, et al. Inverse correlation of each functional status scale of the SF-36 with degree of disease activity in systemic lupus erythematosus (m-SLAM). Joint Bone Spine 2003; 70(5): 348-351.

72. Romero-Diaz J, Isenberg D, and Ramsey-Goldman R. Measures of adult systemic lupus erythematosus: updated version of British Isles lupus assessment group (BILAG 2004), European consensus lupus activity measurements (ECLAM), systemic lupus activity measure, revised (SLAM-R), systemic lupus activity questionnaire for population studies (SLAQ), systemic lupus erythematosus disease activity index 2000 (SLEDAI-2K), and systemic lupus international collaborating Clinics/American College of Rheumatology damage index (SDI). Arthritis Care Res (Hoboken) 2011; 63(11).

73. Mikdashi $\mathrm{J}$ and Nived $\mathrm{O}$. Measuring disease activity in adults with systemic lupus erythematosus: the challenges of administrative burden and responsiveness to patient concerns in clinical research. Arthrit Res Ther 2015; 17(1): 1-10.

74. Chang ER, Abrahamowicz M, Ferland D, et al. Organ manifestations influence differently the responsiveness of 2 lupus disease activity measures, according to patients' or physicians' evaluations of recent lupus activity. The Journal of Rheumatology 2002; 29(11): 2350-2358. 